\title{
QUALIDADE DO MOBILE BANKING NA PERSPECTIVA DOS USUÁRIOS
}

\author{
Jaiarys Capa Bataglin'; \\ Jairo Leonardi de Aguiar ${ }^{2}$
}

\begin{abstract}
This study aims to validate the quality of two services through Mobile Banking, from the perspective of two users, through the importance of carrying out two banking services. The research methodology follows a quantitative, descriptive approach, along with a sample of 95 respondents. Data are collected in a non-probabilistic and convenient manner. The research instrument was adapted from the SERVQUAL model, developed by Parasuraman, Zeithaml, and Berry (1988). The analysis of two data was performed using descriptive statistics techniques, with the aid of the statistical software SPSS, version 20.0. The results indicate that the importance of the quality attributed to the users' hair is greater than the performance of the service provided, in all dimensions of the model, since there are greater gaps in the dimensions Empathy, Reliability, and Responsiveness.
\end{abstract}

Keywords: mobile banking; services; quality; SERVQUAL.

Resumo: O presente estudo buscou avaliar a qualidade dos serviços via Mobile Banking, na perspectiva dos usuários, através da importância e desempenho dos serviços bancários. A metodologia da pesquisa segue uma abordagem quantitativa, com característica descritiva, junto a uma amostra de 95 entrevistados. Os dados foram coletados de forma não probabilística e por conveniência. $O$ instrumento de pesquisa foi adaptado do modelo SERVQUAL, desenvolvido por Parasuraman, Zeithaml e Berry (1988). A análise dos dados foi realizada por meio de técnicas estatísticas descritivas, com o auxílio do software estatístico SPSS, versão 20.0. Os resultados indicam que a importância da qualidade atribuída pelos usuários é maior do que o desempenho do serviço prestado, em todas as dimensões do modelo, com os maiores gaps nas dimensões Empatia, Confiabilidade e Responsividade.

Palavras-chave: mobile banking; serviços, qualidade; SERVQUAL.

\section{INTRODUÇÃO}

\footnotetext{
${ }^{1}$ Graduação em Administração - Universidade Estadual do Oeste do Paraná (Unioeste) Francisco Beltrão - Brasil. Correo electrónico: jaiarys@gmail.com

${ }^{2}$ Graduação em Administração - Universidade Estadual do Oeste do Paraná (Unioeste) Francisco Beltrão - Brasil. Correo electrónico: jairoleonardideaguiar@gmail.com
} 
A busca dos consumidores por serviços bancários mais flexíveis, ágeis e eficientes, têm crescido nos últimos anos. Com a era digital e inserção de novas tecnologias, novos serviços tem sido utilizados, proporcionando maior flexibilidade e aumentando o nível de expectativa dos clientes (Castelli et al., 2017). O Mobile Banking (M-banking) é um exemplo disto, uma nova abordagem no campo dos serviços bancários, por meio do fornecimento de recursos financeiros e serviços bancários via dispositivos móveis (Aghdaie \& Faghani, 2012).

A migração dos serviços bancários - das agências físicas e internet banking - para o $M$ banking, no Brasil, tem decorrido do desenvolvimento tecnológico na área bancária e devido também, à difusão dos smartphones e maior facilidade de acesso aos serviços digitais. Dados da Federação Brasileira de Bancos, evidenciam a alta adesão dos clientes pelo M-banking, dentre os quais estão o aumento na abertura de contas via aplicativo [1,6 milhão] e o volume médio de transações [1,7 bilhão], estimando-se um crescimento de $70 \%$ em comparação aos anos anteriores (Febraban, 2018).

Não obstante, o setor bancário no Brasil, representa um dos setores com maior índice de queixas recebidas por parte dos consumidores (Idec, 2019). Segundo a Febraban (2018), parte dessas reclamações é decorrente da crescente exigência dos clientes por serviços mais ágeis, flexíveis e de maior qualidade. Neste sentido, cabe às instituições bancárias garantir a qualidade e segurança destes serviços (Moller, 2017; Pavarini, Marchetti \& Silva, 2010).

Neste contexto, o presente estudo teve por objetivo compreender a percepção de usuários do $M$-banking, em relação à qualidade dos serviços bancários prestados via aplicativo de celular. Para isso, utilizou o modelo SERVQUAL de avaliação de desempenho, desenvolvido por Parasuraman, Zeithaml e Berry (1988), que busca avaliar a qualidade dos serviços segundo a percepção dos usuários, através de cinco dimensões de qualidade: (1) Tangibilidade, (2) Confiabilidade, (3) Responsividade, (4) Segurança e (5) Empatia. Utilizou uma abordagem quantitativa, com característica descritiva, junto a uma amostra de 95 universitários.

\section{QUALIDADE EM SERVIÇOS}

No sentido mais amplo, qualidade se refere à capacidade de um determinado serviço/produto em atender às expectativas do cliente, ou de superá-la. Assim, pode ser compreendida como a diferença entre as expectativas dos clientes e as percepções do serviço prestado (Parasuraman, 1988).

[Digite aqui] 
A qualidade dos serviços é um dos principais fatores considerados pelo consumidor no momento de avaliar o seu grau de satisfação. Segundo Lovelock e e Wright (2001), os serviços podem ser divididos em dois níveis: (1) serviço desejado - serviço que o cliente espera receber; (2) serviço adequado - nível mínimo de serviço que o clientes aceitará sem ficar insatisfeito. Deste modo, quando o serviço prestado excede às expectativas do cliente, o serviço é percebido como de qualidade excepcional. Porém, a qualidade pode ser insatisfatória quando o serviço prestado não atende às expectativas (Fitzsimmons \& Fitzsimmons, 2005).

Um marco, nessa área, foram os trabalhos publicados por Parasuraman, Zeithaml e Berry (1985, 1988), que tiveram grande influência no desenvolvimento de pesquisas posteriores, na avaliação da qualidade em serviços. O modelo SERVQUAL proposto por Parasuraman, Zeithaml e Berry (1988), procura através de "gaps", identificar lacunas existentes entre a expectativa e a percepção dos usuários, em relação ao serviço prestado. Os gaps foram desenvolvidos com o intuito de auxiliar os gestores na compreensão da fonte dos problemas da qualidade dos serviços, de modo a melhorar a satisfação dos clientes com o serviço prestado.

O modelo é estruturado a partir de cinco dimensões, sendo: (1) tangibilidade, (2) confiabilidade, (3) responsividade (capacidade de resposta), (4) segurança e (5) empatia. Tratase de um valioso instrumento para medir as percepções dos clientes quanto à qualidade do serviço (Fitzsimmons \& Fitzsimmons, 2005; Islam, 2012), sendo a afirmação de expectativa associada a uma afirmação de percepção correspondente. Assim, a qualidade do serviço pode ser compreendida como uma lacuna entre o nível esperado de serviço e as percepções do cliente sobre o nível recebido.

\subsection{MOBILE BANKING}

O M-Banking, é definido como "uma forma de transação bancária realizada via dispositivos móveis" (Amin et al., 2007). Trata-se de um serviço do m-commerce que permite aos clientes acessar contas bancárias e realizar transações financeiras por meio de dispositivos móveis (Rahman, Hasan \& Mia, 2017). Uma das características consideradas únicas dos serviços móveis, diz respeito à onipresença do serviço, característica exclusiva do ambiente móvel (Tojib \& Tsarenko, 2012; Aghdaie \& Faghani, 2012). Além de ser um complemento aos serviços oferecidos pelo sistema bancário, o M-banking oferece uma conveniência a mais, permitindo o gerenciamento do dinheiro, sem o manuseio do mesmo (Karjaluoto, 2002). 
Estudos realizados sobre $M$-banking, tem enfatizado os benefícios trazidos aos clientes, devido à maior flexibilidade e mobilidade das transações, e também, às instituições bancárias por meio da redução de filas no atendimento e custos das agências físicas (Lopes, Caracciolo \& Herrero, 2018). Dentre os fatores que mais tem afetado à adoção destes serviços por parte dos usuários, estão a capacidade do dispositivo móvel e procedimentos de rede, conveniência, segurança, confiabilidade, custo e conhecimento (Deb et al., 2011).

\section{PROCEDIMENTOS METODOLÓGICOS}

A abordagem da pesquisa é quantitativa (Hair Jr et al., 2005), pois buscou mensurar e avaliar a percepção dos usuários em relação à qualidade dos serviços do M-Banking. Com relação ao objetivo, trata-se de uma pesquisa descritiva (Vergara, 2009), pois buscou descrever o perfil socioeconômico dos entrevistados e a percepção de qualidade dos mesmos.

Como estratégia de pesquisa, utilizou-se o método Survey, o qual permite a obtenção de dados e informações sobre um determinado grupo de pessoas, por meio de um questionário estruturado (Hair Jr et al., 2005). A amostra da pesquisa foi constituída por conveniência, assim, foi composta por acadêmicos do curso de Bacharelado em Administração, da Universidade Estadual do Oeste do Paraná, campus Francisco Beltrão/PR. A escolha por tal público se justificou tanto pela acessibilidade, quanto pelo perfil dos usuários em relação ao uso do $M$ Banking (Pavarini, Marchetti \& Silva, 2010). A fim de validar matematicamente o tamanho da amostra, utilizou-se o multiplicador de 5 respondentes por questão, conforme sugerido por Hair Jr et al. (2005). Assim, os dados foram coletados de forma a alcançar um número mínimo de questionários válidos [95].

\subsection{COLETA E ANÁLISE DE DADOS}

Para a coleta dos dados, utilizou-se um questionário estruturado, o qual possibilita medir com maior exatidão a satisfação dos usuários (Hair Jr et al., 2007). O questionário foi adaptado do modelo SERVQUAL, a partir de Parasuraman, Zeithaml e Berry (1988), o qual originalmente contém vinte e dois itens, distribuídos em cinco dimensões da qualidade. A adaptação do questionário ao contexto do M-banking, teve como base outros trabalhos já publicados (Rahman, Hasan \& Mia, 2017; Islam, 2012). 
Após a adaptação do questionário, o mesmo passou pela avaliação de dois especialistas da área de gestão de serviços, assim, três questões foram retiradas do modelo original, dada a redundância das informações - foram excluídas as variáveis 12 [dimensão responsividade], 19 e 20 [dimensão empatia]. O quadro 1, apresenta os atributos avaliados em cada dimensão.

Quadro 1 - Atributos da escala SERVQUAL

\begin{tabular}{|l|l|}
\hline Dimensões & Atributos Avaliados \\
\hline Tangibilidade & $\begin{array}{l}\text { Tecnologia moderna. } \\
\text { Layout grafico atraente. } \\
\text { Facil instalação. } \\
\text { Facilidade de comunicação com suporte. } \\
\text { Oferecer serviços conforme o prometido. } \\
\text { Facilidade e interesse em lidar com os problemas dos clientes. } \\
\text { Oferecer o serviço correto já na primeira vez } \\
\text { Oferecer o produto no prazo prometido. } \\
\text { Indicar possiveis erros dos clientes. }\end{array}$ \\
\hline Responsividade & $\begin{array}{l}\text { Comunicação clara entre o tempo de cada uma das operações no aplicativo. } \\
\text { Prestar pronto atendimento aos clientes. } \\
\text { Agilidade ao responder as demandas dos clientes. }\end{array}$ \\
\hline Segurança & $\begin{array}{l}\text { Metodos de login que inspiram confiança. } \\
\text { Passar segurança ao cliente ao efetuar suas transações. } \\
\text { Metodos de usabilidade consistentemente cordiais. }\end{array}$ \\
\hline Funcionarios preparados para responder questionamentos.
\end{tabular}

Fonte: Adaptado de Parasuraman et al. (1988, p. 207)

O instrumento de avaliação foi composto por duas partes: I) Informações socioeconômica dos entrevistados; II) Itens relacionados à importância e desempenho do serviço prestado. Tais questões foram avaliadas a partir de uma escala Likert de 7 pontos $(1=$ nenhuma importância e 7 = extremamente importante). A Figura 1 ilustra o esquema conceitual da pesquisa. 
Figura 1 - Esquema Conceitual Da Pesquisa

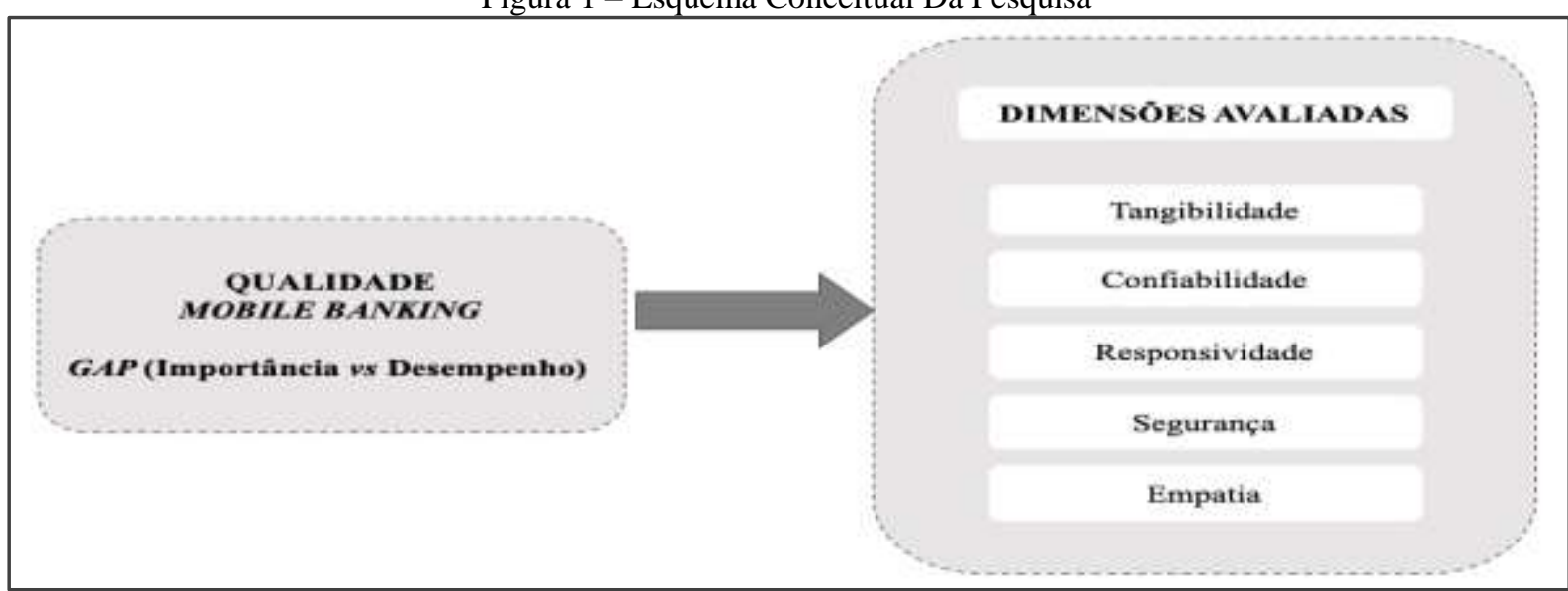

Fonte: Os Autores (2019)

A coleta de dados foi realizada durante a primeira quinzena de outubro de 2019 , com os alunos de graduação do curso de Administração, após a anuência da coordenação do curso e dos professores de cada turma. Os acadêmicos foram orientados a responder inicialmente os itens referentes à mensuração da "importância" e na sequência aos itens referentes à mensuração do "desempenho". Obteve-se um retorno de 103 questionários, no entanto, após os dados serem examinados para verificação da integridade e coerência (Hair Jr et al., 2005), 8 questionários foram eliminados, totalizando 95 questionários válidos.

A análise dos dados foi realizada por meio de técnicas estatísticas, que possibilitam comparar determinadas características entre um conjunto de dados obtidos na pesquisa (Hair Jr et al., 2005). A tabulação e análise dos dados, foi realizada com o auxílio do software estatístico Statistical Package for the Social Sciences (SPSS), versão 22.0. Na primeira etapa, foram utilizadas técnicas da estatística descritiva, por meio do cálculo de frequência simples. $\mathrm{Na}$ sequência, foi calculado o valor médio de cada questão e a média geral de cada dimensão do modelo SERVQUAL. Através do cruzamento das médias (importância versus desempenho), puderam ser observados os gaps de cada dimensão, indicando a satisfação dos clientes com relação a qualidade do serviço.

\section{ANÁLISE E DISCUSSÃO DOS RESULTADOS}

Com relação às características socioeconômicas dos participantes da amostra, os usuários do M-banking, são em sua maioria do sexo feminino [64,2\%], sendo a faixa etária mais representativa da amostra de 20-24 anos [47,4\%], seguido da faixa etária com menos de 20 anos [28,4\%], um público predominantemente jovem.

[Digite aqui] 
Com relação à renda mensal individual, 78,9\% declarou possuir uma renda de até 3 salários mínimos. Sobre a carga horária semanal de trabalho, 63,2\% da amostra declarou trabalhar 40 horas semanais, ou mais. Do restante, 14,7\% apenas estuda e outros 14,7\% trabalham em estágio remunerado. Um percentual menor (6,3\%), trabalha 20 horas semanais e apenas um respondente afirmou "trabalhar eventualmente".

Quando questionados sobre a frequência de utilização do M-banking, 35,5\% afirmaram utilizar o aplicativo de $1-2$ vezes por mês e $29 \%$ de $2-4$ vezes por semana. Outros $25,8 \%$ afirmaram utilizá-lo cotidianamente, o que demonstra o uso desse serviço com bastante frequência. Em relação aos serviços mais utilizados, de acordo com o percentual de respostas, destacam-se serviços como: consulta de saldos e extratos (36,3\%); pagamentos $(28,2 \%)$; transferencias de dinheiro $(15,5 \%)$ e investimentos $(9,0 \%)$. Dentre as instituições financeiras mais utilizadas pelos respondentes, em relação ao M-banking, estão as Cooperativas de Crédito com 29,8\% de respostas. Na sequência, foram indicados os bancos digitais (Nubank, Neon, Next, outros), com 26,6\% e os bancos tradicionais, Banco do Brasil, Bradesco e Santander, totalizando $53,2 \%$ de respostas.

\subsection{IMPORTÂNCIA E DESEMPENHO DO $M-B A N K I N G$}

Nesta seção apresentam-se as análises dos dados da pesquisa relacionados à satisfação com os serviços do M-banking. A pesquisa buscou analisar a percepção do cliente em relação à expectativa e desempenho do serviço. Neste sentido, foram analisadas as médias e desvio padrão de cada dimensão, através da média das respostas de cada indicador. Quanto à confiabilidade do instrumento SERVQUAL, deve-se ressaltar que este modelo de avaliação de serviço tem sido provado consistente e confiável em diversos estudos (Rahman et al., 2017; Aghdaie \& Faghani, 2012).

\subsubsection{Dimensão Tangibilidade}

Para Gronroos (2003), a tangibilidade está relacionada às instalações, equipamentos e materiais usados por uma empresa de serviços. Os indicadores de pesquisa buscaram avaliar a funcionalidade, aparência, instalação e operacionalização do aplicativo. Em relação aos aspectos tangíveis, como pode ser observado na tabela 3, a dimensão obteve uma média alta, 
considerando-se que as respostas foram distribuídas em uma escala de 1 (sem importância) à 7 (extremamente importante).

Tabela 3 - Avaliação da Dimensão Tangibilidade

\begin{tabular}{l|l|l|l|l|l}
\hline \multicolumn{1}{|c|}{ TANGIBILIDADE } & MI & DPI & MD & DPD & GAP \\
\hline $\begin{array}{l}\text { Q1 - O M-banking deve possuir interface (aparência) e } \\
\text { funcionalidades atualizadas. }\end{array}$ & 6,26 &, 775 & 5,88 &, 849 & $-0,38$ \\
\hline $\begin{array}{l}\text { Q2 - A interface (aparência) do aplicativo dever ser visualmente } \\
\text { atrativa. }\end{array}$ & 5,76 &, 953 & 5,63 & 1,032 & $-0,13$ \\
\hline $\begin{array}{l}\text { Q3 - O aplicativo deve ser de fácil instalação e operação. } \\
\text { Q4 - O conteúdo da informação e as instruções devem ser fáceis de } \\
\text { entender. }\end{array}$ & 6,636 &, 602 & 5,95 & 1,014 & $-0,68$ \\
\hline
\end{tabular}

*MI = Média Importância, DPI = Desvio Padrão Importância, MD = Média Desempenho, DPD = Desvio Padrão Desempenho, GAP=Desempenho (Percepção) - Importância (Expectativa). Fonte: Dados da pesquisa, 2019.

A dimensão tangibilidade apresenta um nível alto em termos de expectativa (importância), com média de 6,30 e também em relação ao desempenho efetivo, com média de 5,72 (a segunda melhor média da pesquisa). Embora, a avaliação de desempenho do aplicativo seja inferior à importância, ambas podem ser consideradas altas, indicando satisfação dos usuários em relação aos aspectos de instalação, aparência e funcionalidade do aplicativo.

Um aspecto a ser observado diz respeito ao item Q2 - visual atrativo - que apresentou a menor média na dimensão $(5,76)$ e a segunda menor média na pesquisa. Ramos e Costa (2000), ao investigarem serviços bancários pela internet, também constataram que o atributo "visual atrativo" obteve a pior pontuação, indicando que os usuários atribuem menos importância para este aspecto em relação aos serviços dessa natureza. Já o item Q3 (o aplicativo deve ser de fácil instalação e operação), foi o melhor avaliado na dimensão, tanto em expectativa quanto em desempenho. Segundo a Febraban (2018), a facilidade de instalação e operacionalização dos aplicativos bancários desempenha um papel importante na adoção destes serviços. Tais aspectos tangibilizam a comodidade e flexibilidade na relalção dos usuários com os bancos, com ganhos relevantes em termos de economia de tempo e ausência de filas. Akabane (2016) destaca em seu estudo que o M-banking facilita o acesso, comodidade e autonomia no companhamento e gestão de contas bancárias.

\subsubsection{Dimensão Confiabilidade}


A dimensão confiabilidade expressa se a prestadora oferece aos clientes um serviço correto da primeira vez, sem cometer erros ou falhas e no prazo combinado (Gronroos, 2003). As principais características avaliadas nesta dimensão são, principalmente, a capacidade do aplicativo entregar os serviços, desde a primeira tentativa, conforme o prometido, no tempo estipulado e de forma correta (Tabela 4).

Tabela 4 - Avaliação da Dimensão Confiabilidade

\begin{tabular}{l|l|l|l|l|l}
\hline CONFIABILIDADE & MI & DPI & MD & DPD & GAP \\
\hline $\begin{array}{l}\text { Q5 - Os serviços do M-Banking devem ser realizados conforme o } \\
\text { prometido. }\end{array}$ & 6,62 &, 702 & 5,53 & 1,253 & $-1,09$ \\
\hline $\begin{array}{l}\text { Q6 - O M-banking deve ser capaz de atender as minhas demandas } \\
\text { por serviços bancários. }\end{array}$ & 6,47 &, 861 & 5,78 & 1,204 & $-0,69$ \\
$\begin{array}{l}\text { Q7 - O M-banking deve realizar os serviços corretamente na } \\
\text { primeira tentativa, sem erros. }\end{array}$ & 6,16 &, 891 & 5,34 & 1,456 & $-0,82$ \\
$\begin{array}{l}\text { Q8 - Os serviços do M-banking devem ser realizados dentro do } \\
\text { tempo estipulado. }\end{array}$ & 6,32 &, 775 & 5,58 & 1,276 & $-0,74$ \\
\hline $\begin{array}{l}\text { Q9 - O M-banking deve fornecer informações apropriadas aos } \\
\text { usuários quando ocorre um problema. }\end{array}$ & 6,59 &, 660 & 4,85 & 1,564 & $-1,74$ \\
\hline \begin{tabular}{l} 
MÉDIA DA DIMENSÃO \\
\hline
\end{tabular}
\end{tabular}

*MI = Média Importância, DPI = Desvio Padrão Importância, MD = Média Desempenho, DPD= Desvio Padrão Desempenho, GAP=Desempenho (Percepção) - Importância (Expectativa). Fonte: Dados da pesquisa, 2019.

Todos os itens desta dimensão receberam alto nível de importância, corroborando diversos estudos e pesquisas que apontam a confiabilidade e/ou confiança como um dos fatores críticos na adoção e uso do M-banking (Ramos et al., 2018; Afshan \& Sharif, 2016). Ramos et al. (2018) ao investigarem o efeito da confiança na intenção do uso do M-banking, confirmaram que o construto "confiança" exerce influência significativa no uso pretendido do aplicativo. Individualmente, o item Q5 foi o que obteve o maior nível de importância, indicando uma alta expectativa de que o serviço seja realizado conforme o prometido.

Com relação ao desempenho, foi possível observar que o item Q9 - o M-banking fornece informações apropriadas aos usuários, quando ocorre um problema - obteve a menor avaliação da dimensão $(4,85)$ e a segunda menor avaliação em relação aos demais indicadores da pesquisa. O desafio é aumentar a funcionalidade do aplicativo e de apoio aos clients, garantindo a solução de problemas e disponibilizando informações quanto a eventuais problemas de uso.

Ao estudarem a relação da satisfação com a confiabilidade em bancos, os autores Amin, Isa e Fontaine (2013) observaram que a satisfação com o serviço influencia de forma 
direta e positiva a imagem do banco, que por sua vez influencia a confiabilidade e lealdade dos clientes à estas instituições.

\subsubsection{Dimensão Responsividade}

A responsividade indica o quanto os serviços são oferecidos e entregues de forma rápida e ficaz, avaliando-se, nesse aspecto, a capacidade de resposta da equipe em prover tais serviços (Parasuraman, Zeithaml \& Berry, 1988). No caso do M-banking, os itens Q10, Q11 e Q12 buscou avaliar o tempo de resposta das operações, a disponibilidade de uso dos serviços e a rapidez do serviço do serviço entregue ao cliente (Tabela 5).

Tabela 5 - Avaliação da Dimensão Responsividade

\begin{tabular}{l|l|l|l|l|l}
\hline RESPONSIVIDADE & MI & DPI & MD & DPD & GAP \\
\hline $\begin{array}{l}\text { Q10 - O tempo de cada uma das operações do M-banking, deve } \\
\text { ser claramente comunicado ao usuário. }\end{array}$ & 5,77 & 1,403 & 4,68 & 1,606 & $-1,09$ \\
\hline $\begin{array}{l}\text { Q11 - O M-banking deve oferecer aos usuários serviços e } \\
\text { atendimento on-line 24H. }\end{array}$ & 6,09 & 1,063 & 5,00 & 1,762 & $-1,09$ \\
\hline $\begin{array}{l}\text { Q12 - O M-banking deve oferecer um serviço rápido aos } \\
\text { usuários. }\end{array}$ & 6,39 &, 734 & 5,55 & 1,137 & $-0,84$ \\
\hline MÉDIA DA DIMENSÃO & $\mathbf{6 , 0 8}$ &, 575 & $\mathbf{5 , 0 8}$ & $\mathbf{1 , 5 0 2}$ & $\mathbf{1 , 0 0}$ \\
\hline
\end{tabular}

*MI = Média Importância, DPI = Desvio Padrão Importância, MD = Média Desempenho, DPD= Desvio Padrão Desempenho, GAP=Desempenho (Percepção) - Importância (Expectativa). Fonte: Dados da pesquisa (2019).

A avaliação dessa dimensão apresentou dois dos itens de pesquisa com menor média em relação ao desempenho (Q10 e Q11), com destaque o item Q11, que avalia a disponibilidade e atendimento dos serviços online $24 \mathrm{H}$, com a menor média em relação ao desempenho $(5,00)$. De fato, por se tratar de um serviço móvel (online), pressupõe-se que o serviço esteja disponível 24 horas. A disponibilidade do serviço é indispensável aos serviços digitais, sendo esta uma das vantagens e característica única deste tipo de serviço (Tojib \& Tsarenko, 2012). O item Q11, também apresentou desvio padrão de 1,762, a maior divergência nas respostas em relação aos demais e média 6,09 em relação à expectativa e 5,00 em relação ao desempenho.

O item Q10, está entre os atributos com menor avaliação, tanto em relação à importância $(5,77)$ quanto ao desempenho $(4,68)$. Tal avaliação pode ser decorrência da rapidez das operações bancárias digitais, dispensando a necessidade de comunicação de tempo para a conclusão da operação. Contudo, Afshan e Sharif (2016) afirmam em seu estudo que uma comunicação clara incentiva a confiança dos usuários deste tipo de serviço (M-banking). 


\subsubsection{Dimensão Segurança}

Esta dimensão reflete a segurança e garantia dos serviços prestados, em termos de confiança do usuário em relação ao login no aplicativo, segurança das operações e garantia do sigilo nas informações e operações realizadas no no aplicativo (Gronroos, 2003). Quando comparada às demais dimensões do modelo SERVQUAL, a dimensão segurança apresentou a maior média geral em termos de importância.

Tabela 6 - Avaliação da Dimensão Segurança

\begin{tabular}{l|l|l|l|l|l}
\hline SEGURANÇA & MI & DPI & MD & DPD & GAP \\
\hline $\begin{array}{l}\text { Q13 - As ferramentas do aplicativo (login e todas as operações) } \\
\text { devem inspirar confiança ao usuário. }\end{array}$ & 6,66 &, 709 & 5,99 & 1,135 & $-0,67$ \\
\hline $\begin{array}{l}\text { Q14 - O M-banking deve passar segurança nas transações } \\
\text { realizadas pelos usuários. }\end{array}$ & 6,78 &, 509 & 5,94 & 1,080 & $-0,84$ \\
\hline $\begin{array}{l}\text { Q15 - O sigilo das operações e informações do usuário, no } \\
\text { aplicativo, devem ser garantidas. }\end{array}$ & 6,83 &, 647 & 5,89 & 1,153 & $-0,94$ \\
$\begin{array}{l}\text { Q16 - Deve haver ajuda disponível, quando surgir problemas ao } \\
\text { usar o serviço bancário móvel. }\end{array}$ & 6,26 &, 828 & 5,09 & 1,530 & $-1,17$ \\
\hline \begin{tabular}{l} 
MÉDIA DA DIMENSÃO \\
\hline
\end{tabular}
\end{tabular}

*MI = Média Importância, DPI = Desvio Padrão Importância, MD = Média Desempenho, DPD= Desvio Padrão Desempenho, GAP=Desempenho (Percepção) - Importância (Expectativa). Fonte: Dados da pesquisa (2019).

O item Q13 foi o melhor avaliado nesta dimensão, sendo relacionado ao nível de confiança dos usuários no aplicativo, por exemplo, ao efetuar o login de acesso ao aplicativo. Em relação ao desempenho, obteve uma média de 5,99. Deb et al. (2011), ao estudarem os fatores que afetam a adoção do M-banking em Bangladesh, identificaram a segurança como um dos principais fatores de influência no uso do aplicativo, seguidos de confiabilidade, confidencialidade e flexibilidade, também com influência significativa. Para Afshan e Sharif (2016), é importante que os bancos estipulem e comuniquem de forma clara e objetiva as políticas, garantias e regras que "salvaguardam" a segurança e a confidencialidade dos dados e informações dos usuários dos serviços do M-banking. Em relação ao desempenho, a avaliação da dimensão segurança obteve média geral de 5,73, o que pode indicar uma tendência de os usuários estarem cada vez mais seguros e confiantes em relação à segurança das transações bancárias via aplicativos.

\subsubsection{Dimensão Empatia}

[Digite aqui] 
Esta dimensão avalia o grau de personalização do serviço, conforme a necessidade e perfil do cliente e o quanto é capaz de atender às demandas dos usuários dos serviços.

Tabela 7 - Avaliação da Dimensão Empatia

\begin{tabular}{l|l|l|l|l|l}
\hline EMPATIA & MI & DPI & MD & DPD & GAP \\
\hline $\begin{array}{l}\text { Q17 - O M-banking deve permitir atendimento personalizado em } \\
\text { relação às demandas dos usuários. }\end{array}$ & 5,68 & 1,003 & 5,16 & 1,339 & $-0,52$ \\
\hline $\begin{array}{l}\text { Q18 - O sistema operacional do aplicativo M-banking deve ser } \\
\text { capaz de responder às demandas dos usuários. }\end{array}$ & 6,21 &, 784 & 5,43 & 1,127 & $-0,78$ \\
\hline $\begin{array}{l}\text { Q19 - O aplicativo deve ser funcional e flexível à operação dos } \\
\text { usuários. }\end{array}$ & 6,22 &, 840 & 5,55 & 1,174 & $-0,67$ \\
\hline \begin{tabular}{l} 
MÉDIA DA DIMENSÃO \\
\hline
\end{tabular}
\end{tabular}

*MI = Média Importância, DPI = Desvio Padrão Importância, MD = Média Desempenho, DPD= Desvio Padrão Desempenho, GAP=Desempenho (Percepção) - Importância (Expectativa). Fonte: Dados da pesquisa (2019).

Nesta dimensão, destacam-se a flexibilidade e a funcionalidade do aplicativo, apontados como de maior importância para os usuários do M-banking, como se observa na avaliação do item (Q19), com média (6,22). Para Laukkanen et al. (2007), o M-banking representa uma das mais recentes inovações no setor de serviços bancários possibilitando aos clientes flexibilidade e conveniência e aos bancos significativa redução de custos de operações. A maior conveniência e flexibilidade estão relacionadas à personalização dos serviços, características da dimensão empatia, incentivando à "co-produção" das operações. Neste aspecto, o desenvolvimento de novas tecnologias bancárias tem aperfeiçoando e tornado esses serviços cada vez mais flexíveis e adaptados às necessidades dos clientes (Pavarini, Marchetti \& Silva, 2010).

\subsection{ANÁLISE DOS GAPS IMPORTÂNCIA vs DESEMPENHO}

Esta seção analisa os gaps (lacunas) identificados à partir da avaliação dos atributos, em termos de importância e desempenho dos serviços. O gap é resultado da diferença entre a expectativa e a avaliação do desempenho do serviço entregue. Segundo Lovelock e Wright (2001), a satisfação é resultante da comparação entre o desempenho (resultado percebido) de um produto ou serviço, com as expectativas em relação desempenho esperado. 
Os gaps demonstrados na tabela 8 , foram calculados com o cruzamento das médias de importância e desempenho dos serviços apurados na pesquisa. A tabela 8 apresenta os resultados da avaliação das dimensões do modelo SERVQUAL.

\begin{tabular}{l|c|c|c|c}
\multicolumn{5}{c}{ Tabela 8 - Gaps Importância-Desempenho } \\
\hline Dimensões & $\mathbf{N}^{\mathbf{0}}$ Itens & Média Importância & Média Desempenho & Gap \\
\hline Tangibilidade & 4 & 6,30 & 5,82 & $(-0,48)$ \\
\hline Confiabilidade & 5 & 6,43 & 5,42 & $(-1,01)$ \\
\hline Responsividade & 3 & 6,08 & 5,08 & $(-1,00)$ \\
\hline Segurança & 4 & 6,63 & 5,73 & $(-0,9)$ \\
\hline Empatia & 3 & 6,40 & 5,38 & $(-1,02)$ \\
\hline TOTAL & 19 & 6,36 & 5,48 & $(-0,88)$ \\
\hline
\end{tabular}

Fonte: Dados da pesquisa (2019).

De acordo com os dados apresentados, em nenhuma das dimensões, o desempenho dos serviços do M-banking superou a importância. Os maiores gaps foram identificados nas dimensões Empatia (-1,02) e Confiabilidade (-1,01), sendo o menor gap na dimensão Tangibilidade (-0,48). De outra forma, os referidos Gap podem ser vizualizados no gráfico 2.

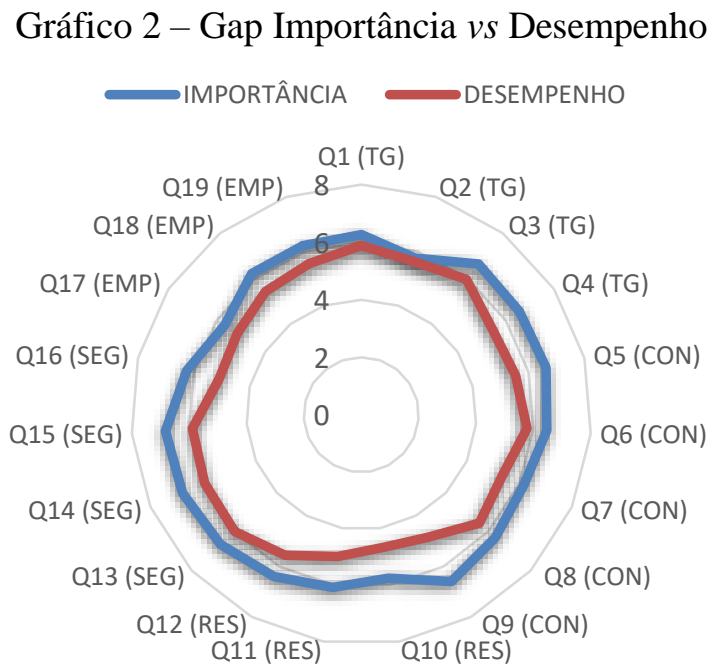

Fonte: Dados da pesquisa (2019)

Conforme o gráfico, é possível visualizar as distâncias (lacunas) entre a importância e o desempenho de cada item avaliado. Distâncias maiores representam os itens que demandam melhorias no serviço entregue - como exemplo, o item Q9 - o M-banking fornece informações apropriadas aos usuários, quando ocorre um problema - com gap $=-1,74$. Para fins gerenciais, 
os gaps são importantes pois permitem identificar lacunas e priorizar as áreas de maior urgência em termos de melhorias na prestação do serviço (Parasuramam, Zeisthaml \& Berry, 1988). Os itens mais demandantes de ação gerencial são, portanto, o Q9 (gap: - 1,74) e o item Q16 (gap: $-1,17)$.

\section{CONSIDERAÇÕES FINAIS}

A pesquisa realizada, buscou avaliar a qualidade dos serviços bancários via Mobile Banking, na perspectiva dos usuários, por meio da relação entre importância e desempenho dos serviços. Após a análise dos dados, foi possível observar que as médias, tanto para a importância quanto para o desempenho foram altas nas 19 questões analisadas, sendo que a média final referente à importância foi de $(6,36)$ - em uma escala de 1 a 7 - e para o desempenho $(5,48)$, com um gap total de $(-0,88)$.

Os itens melhores avaliados em relação à expectativa dos usuários, diz respeito ao sigilo das informações, segurança das transações e confiança na realização das operações, itens da dimensão "Segurança". Já com relação ao desempenho, os itens relacionados à segurança e confiança nas transações, também foram os melhores avaliados, diminuindo os gap desses atributos.

De modo geral, o desempenho do serviço prestado está abaixo da expectativa em todas as dimensões (gaps negativos), neste sentido, a diferença negativa indica que o desempenho do serviço oferecido está abaixo da expectativa do cliente, interferindo diretamente na qualidade percebida. Considerando que a qualidade dos serviços e satisfação dos clientes, se dá pela percepção do usuário quanto ao serviço recebido (Parasuraman, Zeithaml \& Berry, 1988), sugere-se aos provedores de serviços do M-banking, que tenham uma comunicação aprofundada com os usuários, a fim de compreender as suas elevadas expectativas, bem como realizar pesquisas regulares sobre as dimensões de qualidade de serviço, para fazer uma comparação sobre as melhorias implementadas e o efeito dessas melhorias na percepção e satisfação do cliente.

\section{REFERÊNCIAS}

Afshan, S., Arshian, S. (2016). Acceptance of mobile banking framework in Pakistan. Telematics and Informatics, v.33, p.370-387. 
Aghdaie, S.F.A, Faghani, F. (2012). Mobile Banking Service Quality ans Customer Satisfaction (Application of SERVQUAL Model). International Journal Management Business Research, v.2, n.4, pp.351-361.

Akabane, G.K. (2016). Gestão estratégica das tecnologias móveis: evolução, conceitos, modelo de planejamento e tendências. 312p. Jundiaí, Edições Brasil.

Amin, H., Baba, R., Muhammad, M.Z. (2007). An analysis of mobile banking acceptance by Malaysian customers. Sunway Academic Journal, v.4, Universiti Malaysia Sabah.

Amin, M., Isa, Z., Fontaine, R. (2013). Islamic banks:contrasting the drivers of customer satisfaction on image, trust and loyalty of muslim and nonmuslimcustomers in Malaysia. International Journal of Bank Marketing, v.31, n.2, p.79-97.

Castelli, T.M., Longaray, A.A., Lunardi, G.L., Munhoz, P.R.S. Proposta de Instrumento de Avaliação da Satisfação de Usuários de Internet Banking e Mobile Banking: Um Estudo de Caso. (2017). XLI Encontro da ANPAD (EnAnpad). São Paulo, SP.

Deb, S.K., Harun, M.A. and Bhuiyan, M.R.U. (2011). The dimensions affecting the adoption of mobile banking in Bangladesh. Journal of Banking and Financial Services, v. 5,n.1, pp. 97-110.

Febraban (2018). Federação Brasileira de Bancos. Disponível em: < https://portal.febraban.org.br/noticia/3301/pt-br/>. Acesso em: 15 de outubro de 2019.

Fitzsimmons, J.A., Fitzsimmons, M.J. (2005). Administração de Serviços: operações, estratégia e tecnologia da informação. 4 ed. Porto Alegre: Bookman.

Gronroos, C. (2003). Marketing: gerenciamento e serviços. Tradução de Arlete Simille Marques. 2.ed. Rio de Janeiro: Elsevier.

Hair Jr., J. F. Análise multivariada de dados. 5. ed. Porto Alegre: Bookman, 2005.

Idec. (2019). Instituto Brasileiro de Defesa do Consumidor. Disponível em: $<$ https://idec.org.br/idec-na-imprensa/telefonia-e-setor-com-mais-reclamacoes-declientes-em-julho> Acesso em: 16 de setembro de 2019.

Islam, R. M. D. (2012). Application of Servqual Model in Customer Service of Mobile Operators: A Study from the Context of Bangladesh. European Journal of Business and Management, v.4, n.1, pp. 47-54.

Karjaluoto, H. (2002). Selection criteria for a mode of bill payment: Empirical investigationamong Finnish bank customers.International. Journal of Retail \& Distribution Management, v.30, n.6.

Laukkanen, Tommi. (2007). Internet vs mobile banking: Comparing customer value perceptions. Business Process Management Journal, v.13, n.6, p.788-797.

Lovelock, C., Wright, L. (2001). Serviços: marketing e gestão. São Paulo: Saraiva.

Lopes, E.L, Caracciolo, L.L., Herrero, E. (2018). Aceitação do Mobile Banking no Brasil: uma análise por meio do modelo TAM estendido. Teoria e Prática em Administração, v.8, n.1, p.190-221.

Moller, F. (2017). Uso do smartphone por pessoas da terceira idade: a utilização de apps para operações bancárias. Santa Catarina, p. 26.

Parasuraman, A.; Zeithaml, V. A; Berry, L. L. (1985). A conceptual model of servisse quality and its implications for future research. Journal of Marketing, v.49, p.41-50. 
Parasuraman, A.; Zeithaml, V. A; Berry, L. L. (1988). SERVQUAL: A Multiple-Item Scale for Mensuring Consumer Perceptions of Service Quality. Journal of Retailing, v.64, n.1, p.12-40.

Pavarini, S.C.C., Marchetti, R.Z., Silva, W.V. (2010). Mobile Banking: O impacto das características pessoais do consumidor e dos atributos percebidos da inovação na adoção deste serviço. IV Encontro de Marketing da ANPAD (EMA). Florianópolis, SC.

Rahman, A., Hasan, M., Mia, A. (2017. Mobile Banking Service Quality and Costumer Satisfaction in Bangladesh: An Analysis. The Cost and Management, v.45, n.2, p.2532.

Ramos, A.S.M., Costa, F.H.A.R. (2000). Serviços Bancários pela Internet: um Estudo de Caso Integrando a Visão de Competidores e Clientes. $R A C$, v.4, n.3, p.133-154.

Ramos, F.L., Ferreira, J.B., Freitas, A.S., Rodrigues, J.W. (2018). O Efeito da Confiança na Intenção de Uso do m-banking. BBR - Brazilian Business Review, v.15, n.2, p.175-191.

Tojib, D. and Tsarenko, Y. (2012). Post-Adoption Modeling of Advanced Mobile Service Use. Journal of Business Research, v.65, n.7, pp. 922-928.

Vergara, S. C. (2009). Projetos e relatórios de pesquisa em administração. São Paulo: Atlas. 